\title{
PIECEWISE LINEAR NORMAL MICRO-BUNDLES
}

\author{
BY C. T. C. WALL
}

\section{Communicated by J. Milnor, March 4, 1965}

The object of this paper is to gain some information about the unstable piecewise linear groups. The tool that we use for this purpose is the $s$-cobordism theorem (which has been established for piecewise linear manifolds by J. Stallings [9] and D. Barden [1]). All manifolds and micro-bundles in this paper are piecewise linear, unless otherwise specified.

TheOREM 1. Let $M^{m}$ be a compact manifold such that $\pi_{1}(\partial M) \cong \pi_{1} M$ by inclusion, and let $f: K^{k} \rightarrow M^{m}$ be a simple homotopy equivalence of a finite simplicial $k$-complex with $M$. Then if $m \geqq 6, m \geqq 2 k+1$, there is a compact manifold $L$ such that $\pi_{1}(\partial L) \cong \pi_{1} L$, and $L \times I \cong M$. If $m \geqq 7$, $m \geqq 2 k+2, L$ is uniquely determined.

Proof. We first observe that the pair $(M, \partial M)$ is $(m-k-1)$ connected. Indeed, since inclusion induces an isomorphism of tundamental groups, we can use the relative Hurewicz theorem to compute the first nonvanishing relative homotopy group: $\pi_{i}(M, \partial M)$ $\cong \pi_{i}(\tilde{M}, \partial \tilde{M})$ (where $\tilde{M}$ denotes the universal cover), $\pi_{i}(\tilde{M}, \partial \tilde{M})$ $\cong H_{i}(\widetilde{M}, \partial \widetilde{M}) \cong H_{c}^{m-i}(\tilde{M})$, by duality, where $c$ denotes compact cohomology, and $H_{c}^{m-i}(\widetilde{M}) \cong H_{c}^{m-i}(\widetilde{K})$ vanishes for $i<m-k$.

It follows that for $m \geqq 2 k+1, f$ is homotopic to a map $g: K \rightarrow \partial M$. If, now $m \geqq 2 k+2$ we can move $g$ into general position (see e.g. [11, Chapter 6, Theorem 18]) and so suppose it an imbedding. Take a regular neighbourhood $L$ of $g(K)$ in $\partial M$. Then $L$ is a manifold, and the inclusion $L \subset M$ is a simple homotopy equivalence.

If $m=2 k+1, g$ will in general have singularities, transverse selfintersections of $k$-simplexes of $K$. For each such selfintersection $Q=g\left(P_{1}\right)=g\left(P_{2}\right)$, we join $P_{1}$ to $P_{2}$ by a path $\alpha$ in $K$ such that $g(\alpha)$ is a nullhomotopic loop (since $g_{*}: \pi_{1}(K) \rightarrow \pi_{1}(\partial M)$ is onto, this is possible). As $k \geqq 3$, we can now map a disc $D^{2}$ into $\partial M$, with its interior imbedded, and meeting $g(K)$ only in its boundary, which is attached along $g(\alpha)$. Proceeding thus for each selfintersection $Q$, we obtain an imbedding of a complex $K^{\prime}$ simply homotopy-equivalent to $K$; we can then take a regular neighbourhood to obtain $L$, as above. Note in either case that as regular neighbourhood of a subcomplex $K^{\prime}$ of codimension $\geqq 3, L$ has the property $\pi_{1}(\partial L) \cong \pi_{1} L$, for $\partial L$ is a deformation retract of $L-K^{\prime}$. 
Take a collar neighbourhood $\partial L \times I$ of $L$ in $\partial M$ (this is possible since $L$ is a submanifold); let $L^{1}$ be the closure of the complement of $L \cup(\partial L \times I)$ in $\partial M$. We regard $M$ as a cobordism of $L$ and $L^{1}$ : along the 'edge', $\partial L \times I$ is a product cobordism of $\partial L$ and $\partial L^{1}$. Also, the inclusion of $L$ in $M$ is a simple homotopy equivalence. To show that $M$ is an $s$-cobordism, it remains only to check that the inclusion $L^{1} \subset M$ induces an isomorphism of $\pi_{1}$. Now the complement of $L^{1}$ in $\partial M$ is a regular neighbourhood of a $k$-complex, which has codimension $\geqq 3$, so $\pi_{1}\left(L^{1}\right) \cong \pi_{1}(\partial M)$; and by hypothesis, $\pi_{1}(\partial M) \cong \pi_{1}(M)$. Hence $M$ is an $s$-cobordism which along the edge is a product cobordism; by the $s$-cobordism theorem, $M$ is a product: $M \cong L \times I$.

For the proof of uniqueness, we first show that $L$ is in any case the regular neighbourhood of a $k$-complex, given $m \geqq k+4, m \geqq 6$. For by assumption $\pi_{1}(\partial L)=\pi_{1}(L)$; now, as in the proof of existence, $(L, \partial L)$ is $(m-k-2)$-connected. By [10, Theorem 5.5], if $k \geqq 2, L$ has a handle decomposition based on $\partial L$ with no $i$-handles for $i \leqq m-k-2$; the dual decomposition has no $j$-handles for $j>k$, and so $L$ collapses onto a $k$-dimensional spine. If $k=1$, since $m \geqq 6$ we can imbed $K$ in $L$ by a simple homotopy equivalence and take a regular neighbourhood $L^{\prime}$ of the image; by [10, Theorem 6,4] (a variant of the $s$ cobordism theorem) $L$ is diffeomorphic to $L^{\prime}$. We observe that the arguments of [10] can be justified for PL-manifolds by using results from [1] or [9]; we could also use a PL version of the nonstable neighbourhood theorem of Mazur [7, p. 54].

Suppose then $M=L_{1} \times I \cong L_{2} \times I$, and consider the image of $L_{2} \times 0$ in $\partial M$. Since $\partial M$ has dimension $\geqq 2 k+1$, we can deform this to be disjoint from $L_{1} \times 1$, and then a further deformation puts it in the interior of $L_{1} \times 0$. Write $H$ for the closure of $\left(L_{1} \times 0\right)-\left(L_{2} \times 0\right)$; we assert that $H$ is an $s$-cobordism, and hence a product $\partial L_{2} \times I$, so that $L_{1}$ is homeomorphic to $L_{2}$. This can be proved algebraically, or we can use a direct argument by cancellation of handles: for details see Wall $[10$, Theorem 6.4].

We now consider piecewise linear micro-bundles. The basic information on these is contained in Milnor [8]. We write $\epsilon^{r}$ for the trivial micro-bundle with fibre $R^{r}$.

CoROLlaRy 1.1. For any micro-bundle $\xi r$ over $K^{k}$, we can write $\xi^{r}+\epsilon^{2 k} \cong \epsilon^{r}+\eta^{2 k}$ for a suitable micro-bundle $\eta^{2 k}$ of fibre dimension $2 k$. (If $k=2$, replace $2 k$ by 5 .)

Proof. First suppose $k \geqq 3$. Then, as in the theorem, we can imbed some complex simple homotopy-equivalent to $K$ in $R^{2 k}$; thicken it, and call the result $L$. The tangent micro-bundle of $L$ is $\epsilon^{2 k}$. Take the 
total space $M^{1}$ of the micro-bundle induced over $L$ by $\xi^{r}$, and let $M$ be a regular neighbourhood of $L$ in $M^{1}$ : this has tangent micro-bundle $\xi^{r}+\epsilon^{2 k}$. Now by iterating the theorem, we can write $M=N^{2 k} \times D^{r}$, so if $\eta^{2 k}$ is the tangent micro-bundle of $N^{2 k}$, the result follows.

If $k=2$, we replace $R^{4}$ by $R^{5}$, so $L$ has dimension 5 . The argument concludes as before.

CoROLlaRy 1.2. Suppose $\xi^{r}$ and $\eta^{r}$ are stably equivalent micro-bundles over $K^{k}$. Then $\xi^{r}+\epsilon^{2 k} \cong \eta^{r}+\epsilon^{2 k}$. (If $k=2$, replace $2 k$ by 5 .)

Proof. Construct $L$ as above; take regular neighbourhoods $X$ and $Y$ of $L$ in the micro-bundles induced over $L$ by $\xi$ and $\eta$. Since $\xi$ and $\eta$ are stably equivalent, for some $s, X \times D^{s} \cong Y \times D^{s}$. Since $X$ and $Y$ have dimension $2 k+r \geqq 2 k+1$, it follows by iterating the uniqueness part of Theorem 1 that $X$ and $Y$ are PL-homeomorphic. Hence their tangent micro-bundles $\xi^{r}+\epsilon^{2 k}, \eta^{r}+\epsilon^{2 k}$ are equivalent.

REMARK 1. To classify micro-bundles over a 1-complex, it is sufficient to be able to do it over a circle; for this we only need $\pi_{0}\left(\mathrm{PL}_{m}\right)$, which is well known to be $Z_{2}$. Thus if $k=1$, we have $\xi^{r}=\epsilon^{r-1}+\eta^{1}$, and stably equivalent micro-bundles are equivalent.

THEOREM 2. Suppose $K^{k}$ a compact unbounded piecewise linear submanifold of $M^{m}$. Then if $m \geqq 3 k, K^{k}$ has a piecewise linear normal microbundle in $M^{m}$.

Proof. First assume $k \geqq 3$. According to Milnor [8, Theorem 4], for some $n, K^{k}$ has a normal micro-bundle $\xi^{r}$ in $M^{m} \times R^{n}$. By the above corollary, write $\xi^{r}+\epsilon^{2 k}=\epsilon^{r}+\eta^{2 k}$; let $N_{1}$ be a regular neighbourhood of $K$ in the total space of $\eta+\epsilon^{m-3 k}, N_{2}$ a regular neighbourhood of $K$ in $M$. Then $N_{1} \times D^{n}, N_{2} \times D^{n}$ are both regular neighbourhoods of $K$ in $M \times R^{n}$, hence are PL-homeomorphic.

By Theorem 1, if $m \geqq 6, N_{1}$ and $N_{2}$ are PL-homeomorphic. We assert that there is even a PL-homeomorphism inducing the identity on the subcomplex $K$. Granted this, $K$ has a normal micro-bundle in $N_{1}$, hence also in $N_{2}$, and so in $M$.

Write $i_{1}: K \rightarrow N_{1}, i_{2}: K \rightarrow N_{2}$ for the inclusions, and $f: N_{1} \rightarrow N_{2}$ for the PL-homeomorphism constructed above. Then, by the construction of $f, f i_{1} \cong i_{2}$. Since $\operatorname{dim} N_{2}=3 k \geqq 2 k+2$, homotopic imbeddings are isotopic. By the covering isotopy theorem of Hudson and Zeeman [5], since (as $k \geqq 2$ ) the codimension is $\geqq 3$, we can cover the isotopy of $K$ in $N$ by an isotopy $h_{t}$ of $N$. Hence $h_{1} f i_{1}=i_{2}$. The homeomorphism $h_{1} f$ now has the required properties.

In low dimensions we can use a different argument. For if $k \leqq 7$, it follows from smoothing theory (see e.g. [3]) that $N_{2}$ and $K$ admit 
compatible differential structures; if also $2 m \geqq 3 k+3$, by [2, Theorem 2a] the imbedding of $K$ in $N_{2}$ can be approximated by a differentiable imbedding; if finally $2 m \geqq 3 k+4$ these two imbeddings, being homotopic, are PL-isotopic by a theorem of Hudson [4]. Hence $M^{m}$ can be regarded as a smooth manifold with $K^{k}$ as smooth submanifold; as such it has a normal vector bundle and hence a normal PLmicrobundle, according to [6, Theorem 3.2].

AdDendum to Theorem 2 . The result also holds if $k \leqq 7,2 m \geqq 3 k+4$.

This includes those cases of the theorem which were not covered by our first argument.

REMARK 2. The necessity of suspending $\xi$ in the corollaries to Theorem 1-as also the lack of a uniqueness clause in Theorem 2-all stem from our inability, given a complex $K$ and PL-micro-bundle $\xi^{r}$ over $K$, to construct a manifold $M^{r}$ and homotopy equivalence $h: M^{r} \rightarrow K$, such that $h^{*} \xi$ is equivalent to the tangent micro-bundle of $M$. (However large $r$ is, we cannot yet do this.)

Added in proof. Haefliger and the author have now proved a stability theorem for PL-micro-bundles fully analogous to the stability properties of vector bundles, and deduced that Theorem 2 holds for $m \geqq 2 k$.

\section{REFERENCES}

1. D. Barden, The structure of manifolds, Thesis, Cambridge University, 1946.

2. A Haefliger, Differentiable imbeddings, Bull. Amer. Math. Soc. 67 (1961), 109112.

3. M. W. Hirsch, Obstruction theories for smoothing manifolds and maps, Bull. Amer. Math. Soc. 69 (1963), 352-356.

4. J. F. P. Hudson, Combinatorial embeddings and isotopies (to appear).

5. J. F. P. Hudson and E. C. Zeeman, On combinatorial isotopy, Inst. Hautes Etudes Sci. Publ. Math. No. 19, 1964.

6. R. Lashof and M. Rothenberg, Microbundles and smoothing, Topology 3 (1965), 357-388.

7. B. Mazur, Differential topology from the point of view of simple homotopy theory, Inst. Hautes Etudes Sci. Publ. Math. No. 15 (errata in No. 22), 1963.

8. J. Milnor, Microbundles and differentiable structures, Princeton University, 1961, mimeographed.

9. J. Stallings, The s-cobordism theorem (to appear).

10. C. T. C. Wall, Differential topology. IV, Cambridge notes, 1964.

11. E. C. Zeeman, Seminar on combinatorial topology, Inst. Hautes Etudes Sci., Bures-sur-Yvette, 1963, mimeographed.

Mathematical Institute, OXford, England 